\title{
Preparation and characterization of gelatin mediated silver nanoparticles by laser ablation.
}

\begin{abstract}
We successfully prepared colloidal silver nanoparticles (Ag-NPs) using a nanosecond pulsed $\mathrm{Nd}$ :YAG laser, $\lambda=532 \mathrm{~nm}$, with laser fluence of approximately about $0.6 \mathrm{~J} / \mathrm{pulse}$, in an aqueous gelatin solution. The size and optical absorption properties of samples were studied as a function of the laser repetition rates. The results from the UV-vis spectroscopy demonstrated that the mean diameter of Ag-NPs increase with the laser repetition rate increases. The Ag-NPs have mean diameters ranging from approximately $9 \mathrm{~nm}$ to $15 \mathrm{~nm}$. Compared with other preparation methods, this work is clean, rapid, and simple to use.
\end{abstract}

Keyword: Gelatin; Laser ablation; Silver nanoparticles; UV-vis spectroscopy. 